\title{
Improved Heteroepitaxial MBE GaN Growth with a Ga Metal Buffer Layer
}

\author{
Yihwan Kim $^{1}$, Sudhir G. Subramanya ${ }^{1}$, Joachim Krueger ${ }^{1}$, Henrik Siegle ${ }^{1}$, Noad Shapiro ${ }^{1}$, \\ Robert Armitage $^{1}$, Henning Feick ${ }^{1}$, Eicke R. Weber ${ }^{1}$, Christian Kisielowski ${ }^{2}$, Yi Yang ${ }^{3}$, \\ and Franco Cerrina ${ }^{3}$ \\ ${ }^{1}$ Department of Materials Science and Engineering, University of California at Berkeley, and \\ Materials Science Division, Lawrence Berkeley National Laboratory, \\ Berkeley, CA 94720, U.S.A. \\ ${ }^{2}$ National Center for Electron Microscopy, Lawrence Berkeley National Laboratory, \\ Berkeley, CA 94720, U.S.A. \\ ${ }^{3}$ Department of Electrical and Computer Engineering, University of Wisconsin-Madison, \\ Madison, WI 53706, U.S.A.
}

\begin{abstract}
We demonstrate that the use of pure gallium $(\mathrm{Ga})$ as a buffer layer results in improved crystal quality of $\mathrm{GaN}$ epilayers grown by plasma-assisted molecular beam epitaxy on $c$-plane sapphire. The resulting epilayers show electron Hall mobilities as high as $400 \mathrm{~cm}^{2} / \mathrm{Vs}$ at a background carrier concentration of $4 \times 10^{17} \mathrm{~cm}^{-3}$, an outstanding value for an MBE-grown GaN layer on sapphire. Structural properties are also improved; the asymmetric (101) X-ray rocking curve width is drastically reduced with respect to that of the reference $\mathrm{GaN}$ epilayer grown on a low-temperature GaN buffer layer. Nitrided Ga metal layers were investigated for different $\mathrm{Ga}$ deposition time. These layers can be regarded as templates for the subsequent $\mathrm{GaN}$ main layer growth. It was found that there is an optimum Ga metal layer deposition time for improving the electron mobility in the epilayer. Heating of the Ga metal layer to the epilayer growth temperature under nitrogen plasma is found to be sufficient to produce highly oriented $\mathrm{GaN}$ crystals. However, nonuniform surface morphology and incomplete surface coverage were observed after nitridation of comparatively thick Ga metal layers. This is shown to be the reason for the decreasing electron mobility of the epilayers as the Ga metal layer thickness exceeds the optimum value.
\end{abstract}

\section{INTRODUCTION}

Gallium nitride $(\mathrm{GaN})$ and related compounds have attracted considerable academic and commercial interest. This is due to potential application in opto-electronics at short wavelengths, and high-frequency high-power electronics [1,2]. Due to the lack of large area bulk singlecrystalline $\mathrm{GaN}$, epitaxial growth of $\mathrm{GaN}$ has to be done on foreign substrates such as sapphire or SiC. Heteroepitaxial growth on lattice- and thermal-mismatched substrates results in the formation of stress and defects in the GaN epilayer [3]. It is known that the crystal quality of the $\mathrm{GaN}$ epilayer can be improved by introducing a buffer layer between the $\mathrm{GaN}$ epilayer and the substrate $[4,5]$. However, the mechanisms by which the buffer layer relieves stress, and by which the stress relaxation affects defect formation, are not well understood. In a previous study, we found that the composition of the low-temperature GaN (LT-GaN) buffer layer is an important parameter to control stress and material properties of the GaN epilayer [6]. GaN epilayers grown on a Ga-rich LT-GaN buffer layer showed higher crystal quality. This result was attributed to the ability of excess $\mathrm{Ga}$ in the buffer layer to help relax stress at the main layer growth temperature. On the basis of this understanding the present work extends this approach, 
using a Ga metal layer instead of the standard LT-GaN buffer layer in order to maximize the benefits of the excess $\mathrm{Ga}$ in the buffer layer.

\section{GROWTH AND CHARACTERIZATION}

GaN epilayers were grown using a Riber 1000 molecular beam epitaxy (MBE) system. While effusion cells were used to evaporate elemental $\mathrm{Ga}$ and $\mathrm{Si}$, the activated nitrogen (purity $>99.9995 \%$ ) was produced by a Constricted Glow Discharge (CGD) plasma source developed at Lawrence Berkeley National Laboratory. A liquid nitrogen cryoshroud was used during growth to obtain a base pressure in the chamber of $\sim 2 \times 10^{-10}$ Torr. Titanium films (800 $\mathrm{nm}$ thick) were coated on the backs of the $10 \times 11 \mathrm{~mm}^{2} \mathrm{c}$-plane sapphire substrates in order to increase heat absorption by radiation from the tungsten filament heater. The substrate temperature was monitored using a pyrometer. Prior to growth, the substrates were degreased by boiling in acetone and ethyl alcohol for 5 minutes each and dried with flowing nitrogen gas.

A schematic diagram of the growth procedure is given in Figure 1. It is similar to the standard two-step growth with LT-GaN, with the exception that there is no nitrogen plasma during the buffer layer deposition. First, the substrates were exposed to nitrogen plasma for nitridation for 10 minutes at $700{ }^{\circ} \mathrm{C}$. Subsequently, thin pure metallic Ga buffer layers were deposited at $500^{\circ} \mathrm{C}$. The deposition time was varied between $2.5 \mathrm{~min}$ and $10 \mathrm{~min}$. Then, the Ga metal layers were heated up to the main layer growth temperature, $725^{\circ} \mathrm{C}$, under nitrogen plasma. Finally, the main layer was grown at $725^{\circ} \mathrm{C}$. The main layer growth rate was approximately 0.5 $\mu \mathrm{m} / \mathrm{hr}$. For each buffer layer deposition time, main layers were grown for $0 \mathrm{~min}, 5 \mathrm{~min}, 30 \mathrm{~min}$, and $4 \mathrm{hr}$, resulting in 16 samples as indicated in Fig. 1. This enables us to systematically investigate the growth mechanism. The nitridation of the Ga metal layer takes place during the heating to the main layer growth temperature under nitrogen plasma. For studies of the nitrided Ga layers without main layer growth, the samples were rapidly cooled to room temperature after reaching the main layer growth temperature.

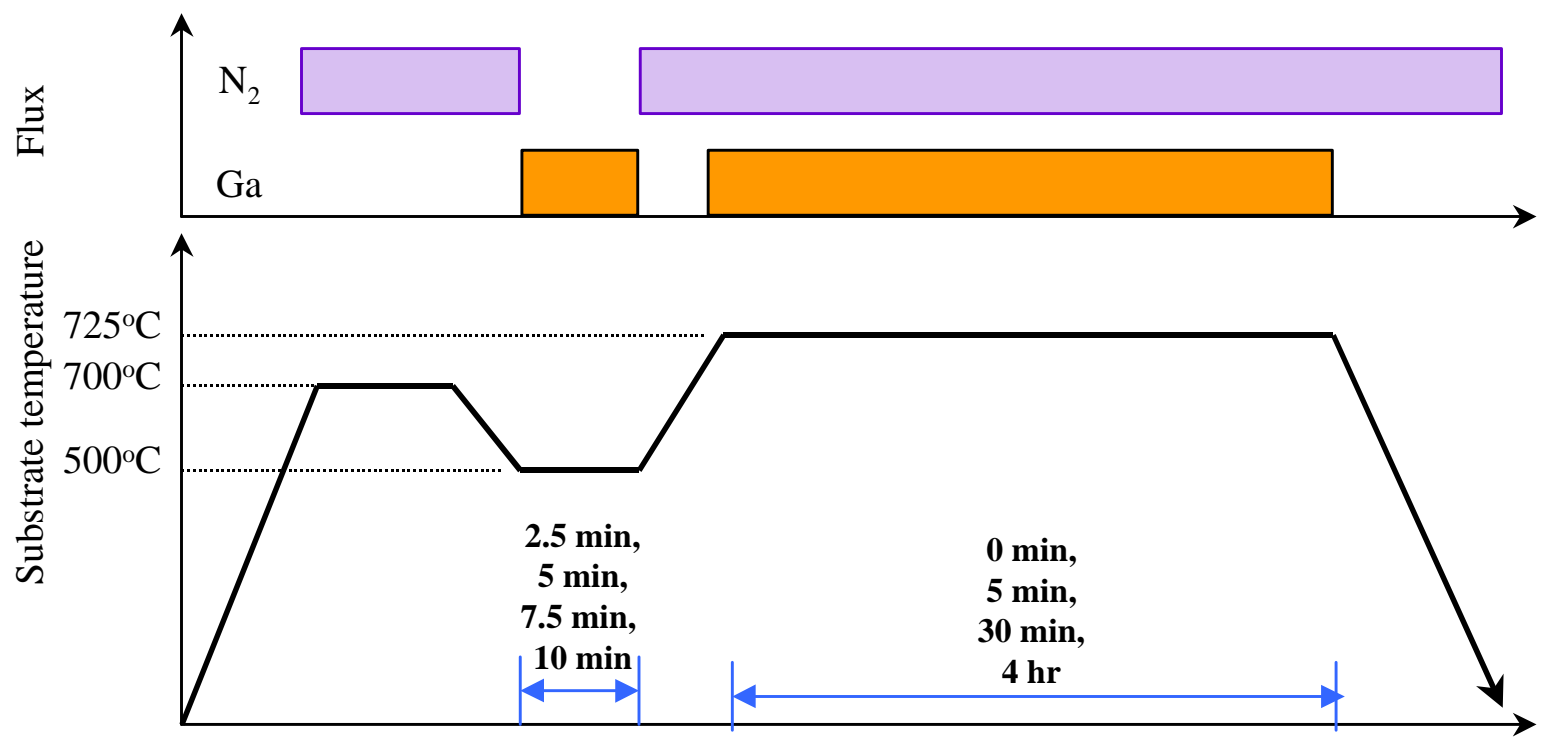

Figure 1. Schematic diagram of the growth procedure with a Ga metal buffer layer. 
Free carrier concentrations and mobilities were measured by Hall effect at room temperature. The crystalline quality of the $\mathrm{GaN}$ main layers and the nitrided Ga layers was assessed by X-ray diffraction taking the full width at half maximum (FWHM) of the symmetric (002) and the asymmetric (101) rocking curves. Cross-sectional transmission electron microscopy (TEM) was used to investigate the microstructure of selected films. The surface morphology was evaluated by contact mode atomic force microscopy (AFM). In order to detect metallic $\mathrm{Ga}$ in the nitrided Ga metal layer, synchrotron radiation photoemission spectroscopy on the undulator Beamline 12 at the Advanced Light Source (ALS) at the Lawrence Berkeley National Laboratory was used.

\section{RESULTS AND DISCUSSION}

\section{The nitrided Ga layer for different Ga metal buffer layer deposition time}

Figure 2 shows x-ray diffraction data from the nitrided Ga layers. The phi scan along the (002) axis shows two peaks separated by 180 degrees, and the phi scan along the (101) axis shows six peaks separated by 60 degrees. This means that the Ga metal is converted into highly oriented GaN crystals during heating to the main layer growth temperature under nitrogen plasma. The FWHM of the (002) x-ray rocking curves of the nitrided Ga layer have a range of 18 30 arcmin, and the FWHM of the (101) x-ray rocking curve is about 40 arcmin.

In order to measure excess $\mathrm{Ga}$ in the nitrided Ga layers, we carried out a synchrotron radiation photoemission study. From the measured photoemission spectra with $130 \mathrm{eV}$ photon energy, two peaks representing Ga 3d can be resolved, as shown in Figure 3(a). The peak at lower kinetic energy corresponds to $\mathrm{Ga} 3 \mathrm{~d}$ in $\mathrm{GaN}$, and the higher kinetic energy peak corresponds to $\mathrm{Ga} 3 \mathrm{~d}$ in metallic $\mathrm{Ga}$ [7]. Evidently, excess Ga remains in the nitrided Ga layers in metallic form. From the measured photoemission spectra, the peak-area ratio of $\mathrm{Ga} 3 \mathrm{~d}$ in $\mathrm{Ga}$ metal to $\mathrm{Ga} 3 \mathrm{~d}$ in $\mathrm{GaN}$ was calculated and plotted as a function of Ga metal deposition time, as shown in Figure 3(b). As the Ga metal deposition time increases, the Ga metal fraction increases in the nitrided $\mathrm{Ga}$ layer. This result is expected since all $\mathrm{Ga}$ metal layers were exposed to nitrogen plasma for the same amount of time. Therefore, the main layer growth on the thicker nitrided Ga metal layer, containing more $\mathrm{Ga}$ in metallic form, is expected to result in the higher crystal quality according to the hypothesis in our previous study [6].
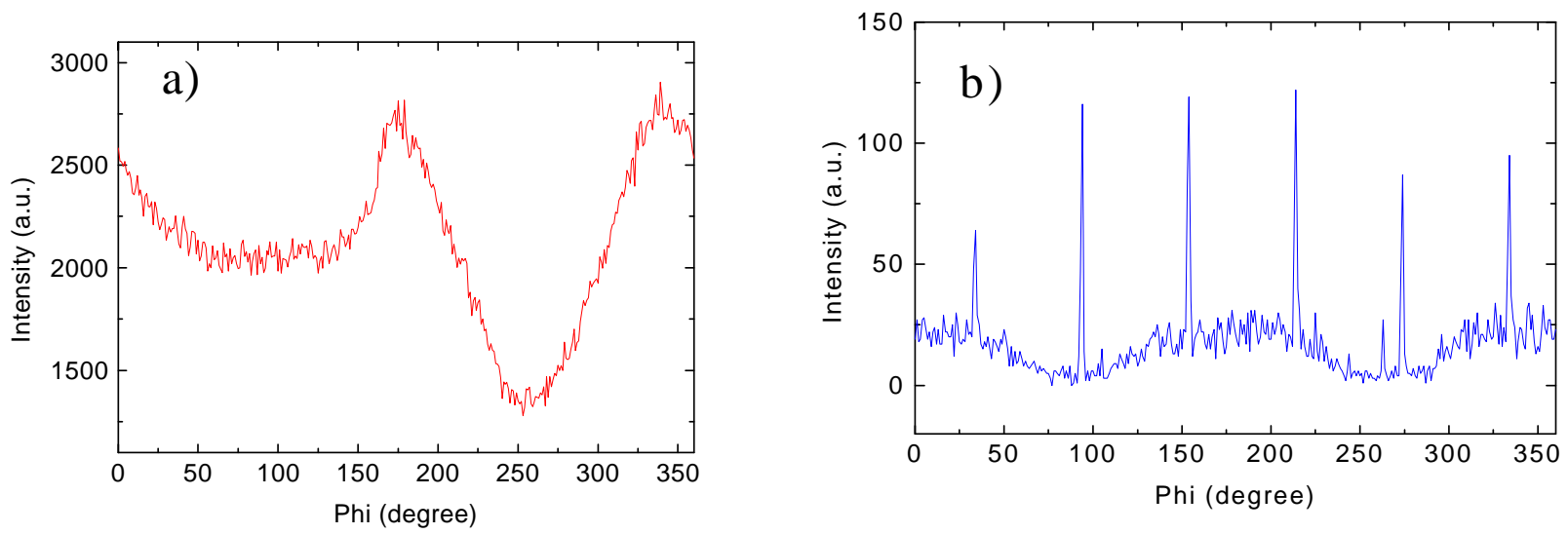

Figure 2. Phi scans of the nitrided Ga metal layer along a) the (002) axis and b) the (101) axis. 

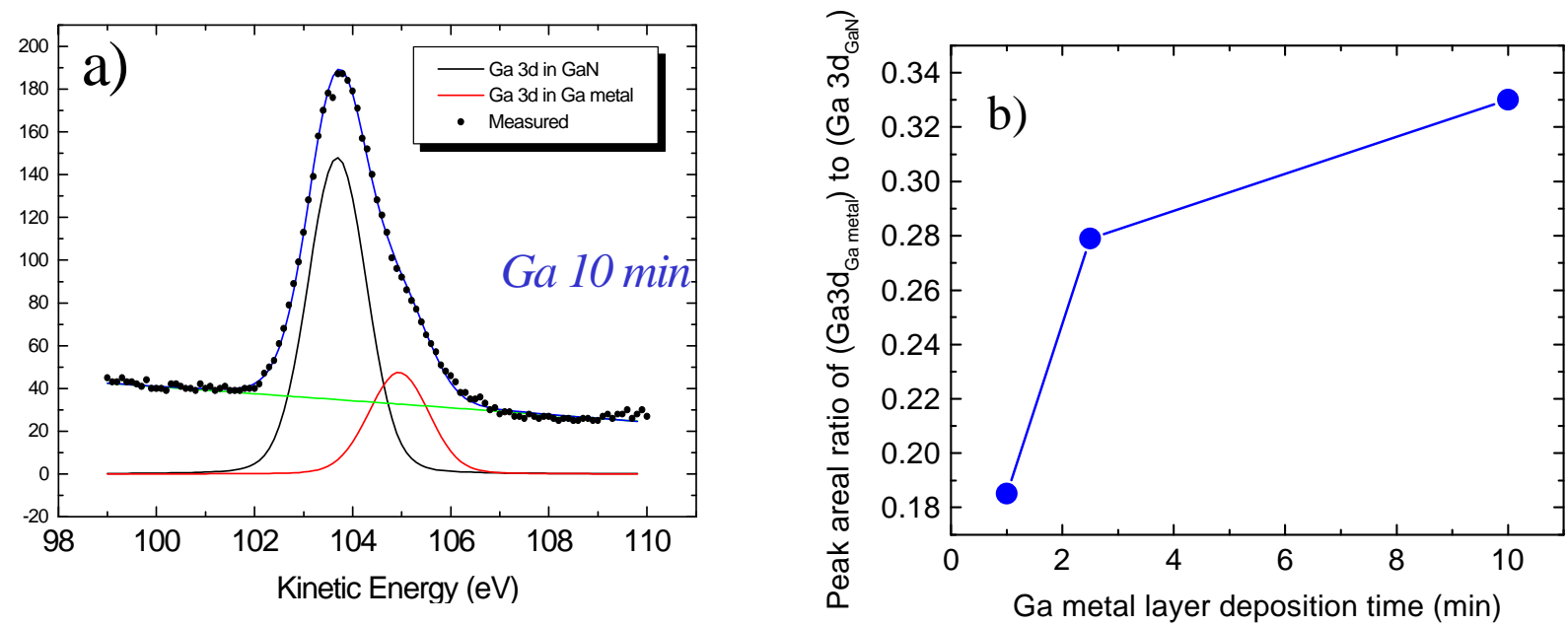

Figure 3. a) A photoemission spectrum from the nitrided Ga layer deposited for 10 min. Two Ga $3 d$ peaks can be resolved. b) variation of the peak area ratio of Ga3d from the Ga metal to Ga $3 d$ from GaN as a function of the deposition time of the Ga metal buffer layer.

\section{GaN main layers grown for 4 hrs with different Ga metal buffer layer deposition time}

Figure 4 shows the electron mobility and carrier concentration of the GaN main layers as a function of the $\mathrm{Ga}$ metal layer deposition time. The electron mobility goes through a maximum of $400 \mathrm{~cm}^{2} / \mathrm{Vs}$ for 5 minutes $\mathrm{Ga}$ metal deposition time. The mobility value is outstandingly high compared with other reported values for MBE-grown GaN epilayers. This value is also higher than the $320 \mathrm{~cm}^{2} / \mathrm{Vs}$ found for GaN epilayers grown with optimized LT-GaN buffer layers in our previous studies. The carrier concentration was found to be $4 \times 10^{17} \mathrm{~cm}^{-3}$, independent of the Ga metal deposition time. Thus, impurity scattering cannot account for the change in mobility. The full widths at half maximum (FWHM) of symmetric (002) and asymmetric (101) x-ray rocking curves of the $\mathrm{GaN}$ main epilayers were also measured. It is known that the FWHM of the symmetric (002) and asymmetric (101) rocking curves are indicators of the tilt and twist disorder of the crystal, respectively [8]. Especially, the asymmetric rocking curve width is a more reliable indicator of the structural quality of the epilayer [9]. All $\mathrm{GaN}$ epilayers in this study showed an asymmetric FWHM of less than 13 arcmin and a symmetric FWHM of less than 7 arcmin. The FWHM of the (101) rocking curve in particular is significantly reduced compared with the 27 arcmin we observed for $\mathrm{GaN}$ epilayers on LT-GaN buffer layers.

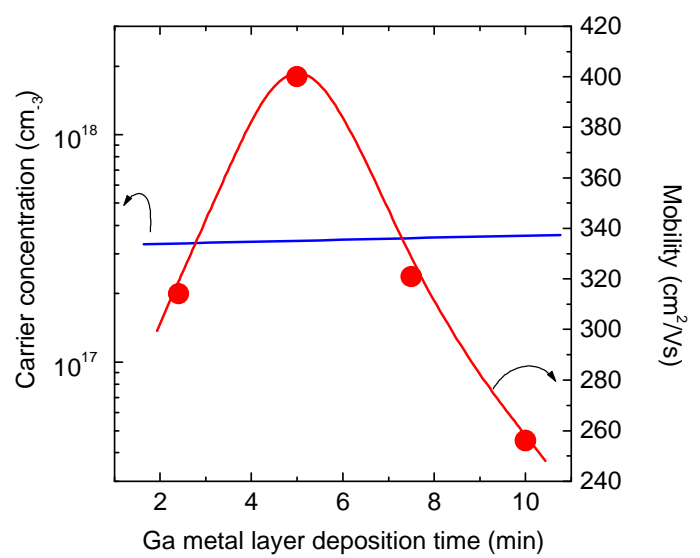

Figure 4. Electron mobility and carrier concentration of the GaN epilayer as a function of the Ga metal buffer layer deposition time. 

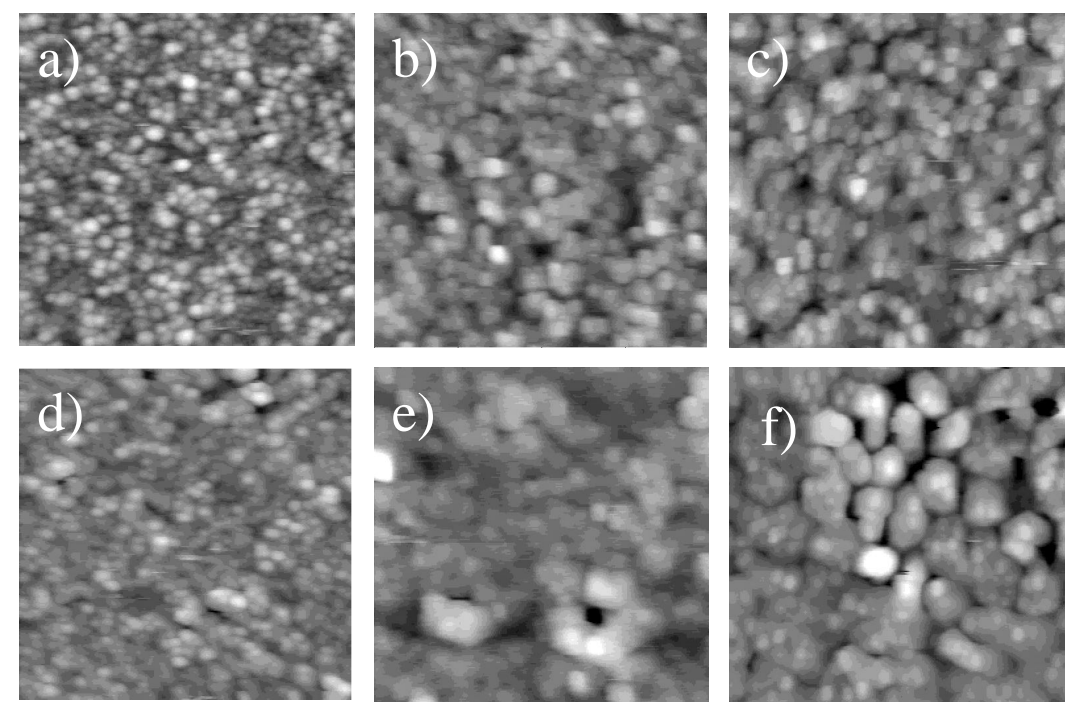

Figure 5. AFM images $(2 \mu m \times 2$ $\mu \mathrm{m})$ of the nitrided Ga layers ( $(a)$ and $(d))$, GaN epilayers grown for 5 min on the nitrided Ga layers ( $(b)$ and (e), and GaN epilayers grown for 30 min on the nitrided Ga layers ((c) and $(f)) .(a),(b)$, and $(c)$ are for the 5 min Ga metal layer deposition time. $(d),(e)$, and $(f)$ are for the $10 \mathrm{~min} G a$ metal layer deposition time.

\section{Optimum Ga metal deposition time for the electron mobility in of the GaN main layer}

As shown in Figure 4, there is an optimum Ga metal deposition time for the Ga metal in terms of the mobility of the GaN main layer. This can be explained by the surface morphology of the nitrided $\mathrm{Ga}$ layer and the subsequently grown $\mathrm{GaN}$ main layers. Figure 5 shows the surface morphology of the nitrided Ga layers and the GaN main layers for $5 \mathrm{~min}$ and $10 \mathrm{~min} \mathrm{Ga}$ metal deposition time. A nonuniform surface morphology is observed in the nitrided Ga layer for $10 \mathrm{~min} \mathrm{Ga} \mathrm{metal} \mathrm{deposition} \mathrm{time,} \mathrm{and} \mathrm{this} \mathrm{is} \mathrm{replicated} \mathrm{by} \mathrm{the} \mathrm{subsequent} \mathrm{main} \mathrm{layers,} \mathrm{as}$ shown in Figures 5 (d), (e), and (f).

Incomplete substrate coverage was also observed with cross-sectional TEM (Figure 6). Deep trenches, also observed on the previous AFM pictures, are seen in Figure 6(a). Figure 6 (c) shows a higher magnification of the nitrided $10 \mathrm{~min}$ Ga metal layer. An exposed substrate region is seen between $\mathrm{GaN}$ islands. It was confirmed that this is not caused by ion beam damage during TEM specimen preparation. For comparison, Figure 6(b) shows a cross section of the thinner nitrided Ga metal layer, displaying complete coverage. It is argued that this nonuniform surface morphology in the thickest nitrided Ga metal layer is the reason for the reduced electron mobility of the main layer grown on it.

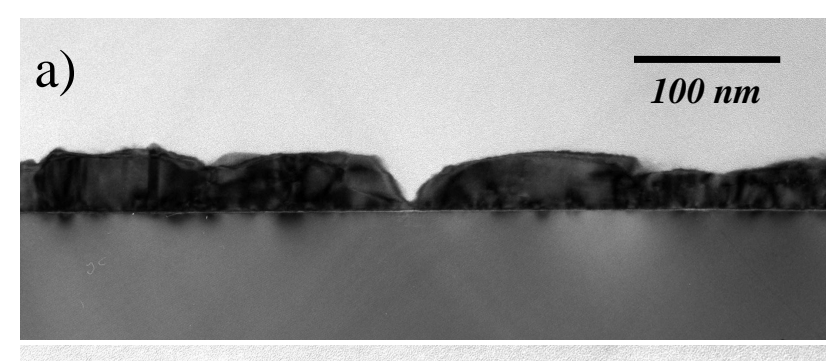

b)

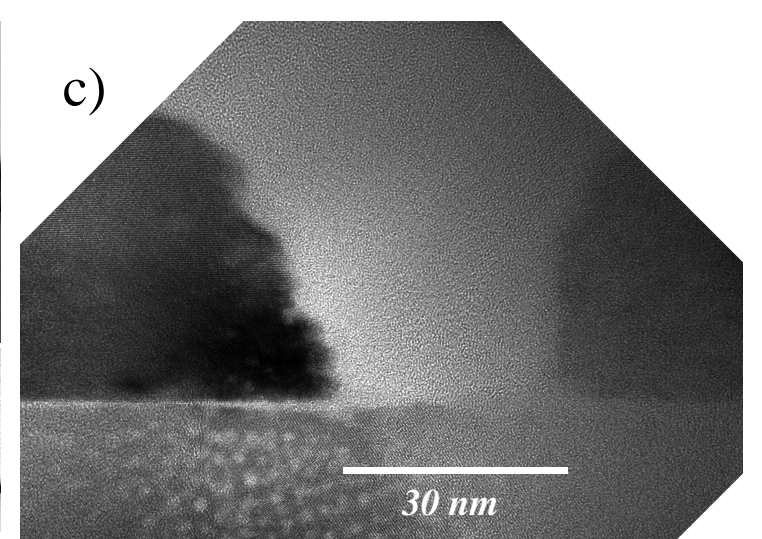

Figure 6. Cross sectional transmission electron micrographs of a) the nitrided 10 min Ga metal layer and b) the nitrided 2.5 min Ga metal layer; c) shows the exposed substrate between GaN islands from the nitrided 10 min Ga metal layer with higher magnification. 


\section{CONCLUSIONS}

We have demonstrated that improved crystal quality of GaN epilayers grown heteroepitaxially on sapphire can be obtained using a pure metallic Ga buffer layer instead of a standard LT-GaN buffer layer. Significantly narrower x-ray rocking curve widths and outstandingly higher electron mobilities were observed. Exposure of the Ga metal layer to nitrogen plasma during heating to the main layer growth temperature results in highly oriented $\mathrm{GaN}$ crystals. It was also observed that excess Ga remains in metallic form in the nitrided $\mathrm{Ga}$ layer and that its fraction was increased with thicker nitrided Ga layer. In this growth procedure there is an optimum Ga metal thickness for improving the mobility in the GaN main layer. The reason was found to be that the thickest Ga metal layer causes nonuniform surface morphology after nitridation and this nonuniformity was replicated by the subsequent main layer growth.

\section{ACKNOWLEDGMENTS}

This work was supported by the Office of Science, Basic Energy Sciences, Division of Materials Sciences, of the U. S. Department of Energy under contract No.DE-AC03-76SF00098. The $\mathrm{x}$-ray diffractometer is part of the Integrated Materials Laboratory at the University of California at Berkeley, funded by the National Science Foundation. The use of the TEM facilities at the National Center for Electron Microscopy at Lawrence Berkeley National Laboratory is greatly appreciated. Y. K. thanks Dr. J. P. Ahn for helpful discussions on the TEM study.

\section{REFERENCES}

1. S. Nakamura, M. Senoh, N. Iwasa, and S. Nagahama, Appl. Phys. Lett. 67, 1868 (1995).

2. M. A. Kahn, A. Bhattarai, J. N. Kuzina, D. T. Olsen, Appl. Phys. Lett. 63,1214 (1993).

3. C. Kisielowski, J. Krüger, S. Ruvimov, T. Suski, J.W. Ager III, E. Jones, Z. Liliental-Weber, H. Fujii, M. Rubin, E. R. Weber, M. D. Bremser, and R. F. Davis, Phys. Rev. B 54, 17745 (1996).

4. H. Amano, N. Sawaki, I. Akasaki, and Y. Toyoda, Appl. Phys. Lett. 48, 353 (1986).

5. S. Nakamura, Jpn. J. Appl. Phys. 30, L1705 (1991).

6. Y.Kim, Sudhir G.S., H. Siegle, J. Krüger, P. Perlin, E. R. Weber, S. Ruvimov, and Z. LilientalWeber, submitted to J. Appl. Phys.

7. R. Carin, J. P. Deville, and J. Werckmann, Surface and Interface Analysis, 16, 65 (1990).

8. F. A. Ponce, MRS Bull. 22, 51 (1997).

9. B. Heying, X. H. Wu, S. Keller, Y. Li, D. Kapolnek, B. P. Keller, S. P. DenBaars, and J. S. Speck, Appl. Phys. Lett. 68, 643 (1996). 